\title{
Extension de la pourriture molle du céleri branche en maraîchage périurbain de Yaoundé (Cameroun)*
}

\author{
Serge Simon ${ }^{1}$ \\ Ghislain Mboueda Kougang ${ }^{2}$ \\ Éric Nanga Bikoula ${ }^{2}$ \\ ${ }^{1}$ Cirad \\ Département Persyst \\ TA B-103/PS4 \\ Boulevard de la Lironde \\ 34398 Montpellier cedex 5 \\ France \\ <serge.simon@cirad.fr> \\ 2 Université de Dschang \\ Faculté d'agronomie et des sciences agricoles \\ BP 96 \\ Dschang \\ Cameroun \\ $<$ mbouedaghislain@yahoo.fr> \\ $<$ m_nanga@yahoo.fr>
}

\begin{abstract}
Résumé
Le céleri branche, Apium graveolens var. dulce, est un légume-feuille qui domine les cultures maraîchères du bas-fond de Nkolondom, principal pourvoyeur en légumes de Yaoundé (Cameroun). Cultivé comme les légumes-feuilles traditionnels, le céleri branche est toutefois menacé depuis trois ans par l'extension d'une pourriture molle qui réduit d'un tiers la durée de son cycle cultural. La bactérie Pectobacterium carotovorum, anciennement appelée Erwinia carotovora, ayant été identifiée comme agent causal, le mode local de récolte par prélèvements échelonnés de feuilles a été soupçonné de favoriser cette maladie par les blessures induites sur la tige. Le mode de conduite local de la culture a donc été comparé à l'itinéraire technique conventionnel de région tempérée (région d'origine du céleri), qui diffère par le mode de semis, de plantation, et de récolte. Les résultats montrent que le mode de récolte échelonnée ne favorise pas l'extension de la maladie, qu'il procure les meilleurs rendements, et qu'il est bien adapté au marché local. Finalement, l'extension récente de $P$. carotovorum pourrait être liée au changement concomitant de la variété de céleri imposée par les importateurs de semences, ce qui souligne la fragilité d'une filière maraîchère encore peu organisée.
\end{abstract}

Mots clés : agriculture périurbaine ; Apium graveolens ; Cameroun ; culture maraîchère ; Erwinia carotovora.

Thèmes : productions végétales ; systèmes agraires.

\section{Abstract \\ Extension of soft decay in celery in periurban horticulture in Yaoundé (Cameroon)}

Celery, Apium graveolens var. dulce, is the most important leafy vegetable crop in the Nkolondom inland valley, which supplies the majority of vegetables destined for sale in Yaoundé (Cameroon). Celery is a traditional leafy vegetable, but in recent years the crop has been threatened by the extension of soft decay which reduces the period during which it can be grown by one third. As the bacterium Pectobacterium carotovorum, formerly called Erwinia carotovora, was identified as the causal agent, the harvest technique, which involves the phased removal of individual leaves, was suspected of facilitating the spread of the disease through scars left on the stems. Local cultivation practices were compared with standard practices in temperate regions (where celery originated), which differ in sowing, planting, and harvesting techniques. The results showed, firstly, that the extension of the disease was not due to the local harvesting technique, which is well adapted to the local market, and secondly, that better yields are obtained using the local technique. The recent extension of the disease could be due to a change in the variety of celery, which was introduced at the same time as the extension of the disease became apparent. The new variety was planted because of difficulties in importing celery seeds, thus highlighting the inherent risk of any poorly organized innovation in a vegetable sector.

Key words: Apium graveolens; Cameroon; Erwinia carotovora; suburban agriculture; vegetable growing.

Subjects: farming systems; vegetal productions.
*Pour citer cet article: Simon S, Mboueda Kougang G, Nanga Bikoula E. Extension de la pourriture molle du céleri branche en maraîchage périurbain de Yaoundé (Cameroun). Cah Agric 2010 ; 19 : 460-4 ; doi : 10.1684/agr.2010.0435 
$\mathrm{D}$ eux cent sept espèces de légumes-feuilles, dont le céleri branche (Apium graveolens var. dulce), ont été recensées en Afrique (Kahane et al., 2005). Outre leur apport nutritionnel important (Van Rensburg et al., 2004), ces cultures jouent un rôle majeur au Cameroun où elles occupent la troisième position en valeur après l'oignon et la tomate (Temple et al., 2008). Le céleri branche, introduit dans les années 1970 au Cameroun, domine le bas-fond maraîcher périurbain de Nkolondom, cultivé dans 400 exploitations familiales et principal pourvoyeur de Yaoundé en légumes. Or, depuis trois ans, l'extension d'une pourriture molle met en péril cette culture pivot des assolements. Cette note rapporte les résultats de la recherche de l'agent causal ainsi que des facteurs techniques qui pourraient en expliquer l'extension ; elle met également en lumière l'intérêt des agriculteurs à cultiver le céleri de la même manière que les légumes-feuilles traditionnels.

\section{Matériel et méthode}

\section{Itinéraire cultural}

Le bas-fond de Nkolondom (30 50' Nord, $11^{\circ} 30^{\prime}$ Est, altitude $760 \mathrm{~m}$ ) bénéficie d'un climat subéquatorial de type guinéen à pluviométrie bimodale $(1290 \mathrm{~mm} / \mathrm{an}$, température moyenne $25^{\circ} \mathrm{C}$ ).

Les agriculteurs achètent les semences importées de céleri branche, car il n'existe pas de production locale de graines pour cette plante bisannuelle. L'itinéraire cultural du céleri branche est identique à celui des légumes-feuilles traditionnels. Le semis est effectué à la volée à la dose de 10 à $20 \mathrm{~g} / \mathrm{m}^{2}$ (alors que l'itinéraire conventionnel de région tempérée recommande $1 \mathrm{~g} / \mathrm{m}^{2}$ en lignes espacées de $10 \mathrm{~cm}$ ). Les plants sont repiqués précocement (stade 2-3 feuilles) à la densité de 30 plants $/ \mathrm{m}^{2}$, souvent complantés en quinconce avec un autre légume-feuille à cycle court nécessitant peu d'intrants. Les revenus issus de la récolte de la culture associée servent ainsi à acheter les intrants du céleri branche. L'irrigation manuelle sur frondaison $\left(6 \mathrm{~L} / \mathrm{m}^{2}\right)$ est pratiquée un jour sur deux. La fertilisation n'intervient qu'après la dernière récolte de la culture associée: toutes les deux semaines et à la surface du sol, sont épandus $4 \mathrm{~kg} / \mathrm{m}^{2}$ de fumier de fientes de volailles ou $50 \mathrm{~g} / \mathrm{m}^{2}$ d'un engrais complexe de formulation $20 \mathrm{~N}-10 \mathrm{P}-10 \mathrm{~K}$.

La protection phytosanitaire concerne les deux principaux bioagresseurs : la septoriose (maladie fongique du feuillage due à Septoria apiicola) et la mouche mineuse (Lyriomiza sp.) qui creuse des galeries dans les folioles. Les pulvérisations associant un fongicide et un insecticide de synthèse sont hebdomadaires en début de culture, puis faites après chaque récolte (tous les 10 jours).

Les récoltes débutent en moyenne 10 semaines après plantation et consistent à prélever sur chaque plante une ou deux feuilles externes (figures 1A, 1B). Au fur et à mesure des récoltes, se forme une tige aérienne qui porte les cicatrices des pétioles des feuilles récoltées (figure 1C).

\section{Identification de l'agent causal de la pourriture molle}

Aux dires des producteurs, la pourriture molle touchait auparavant moins de $5 \%$ des plants d'une parcelle mais, depuis trois ans, elle détruit plus de la moitié des plants en quelques mois. De ce fait, la durée du cycle cultural est passée de 7 à 4-5 mois. Les pourritures observées ont d'abord été attribuées à des carences en bore ou en calcium, auxquelles le céleri branche est sensible (Bouzo et al., 2007). Or, en 2007, les analyses de sols qui ont été faites pour tester cette première hypothèse, ont révélé des teneurs moyennes de $2,5 \mathrm{meq} / 100 \mathrm{~g}$ de calcium et de $2,9 \mathrm{mg} / \mathrm{kg}$ de bore, supérieures aux seuils de carence, respectivement de $1,5 \mathrm{meq} / 100 \mathrm{~g}$ et $2 \mathrm{mg} / \mathrm{kg}$. Dans le même temps, les analyses microbiologiques de plants présentant les premiers

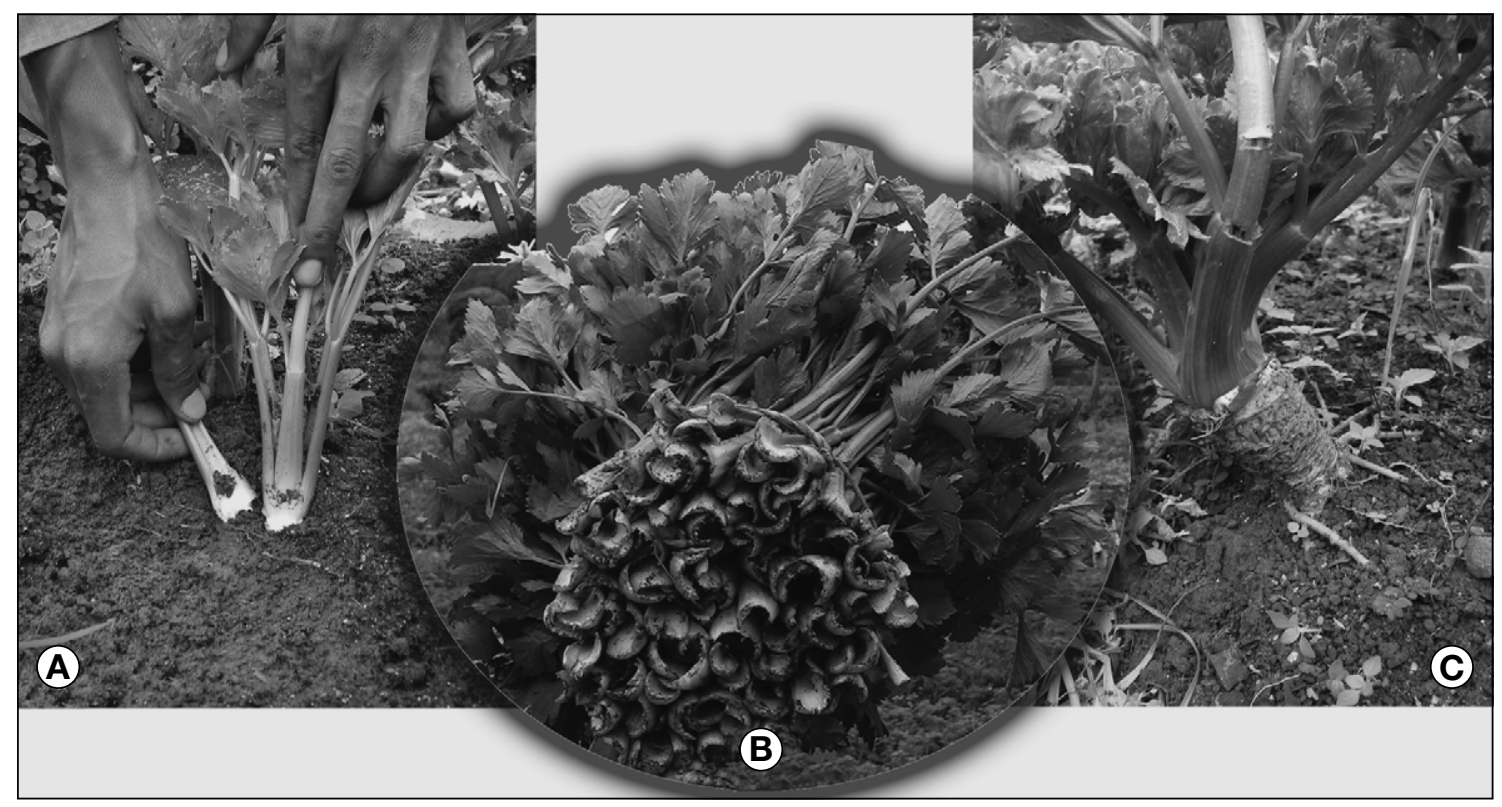

Figure 1. Récolte traditionnelle du céleri branche dans le bas-fond de Nkolondom (Yaoundé).

Figure 1. Local harvesting of celery in Yaoundé.

A) prélèvement échelonné de feuilles individuelles ; B) conditionnement en bottes de $6 \mathrm{~kg}$; C) tige aérienne portant les cicatrices de pétioles. 
symptômes de pourriture molle ont révélé le véritable agent causal, Pectobacterium carotovorum, connu jusqu'en 2006 sous l'appellation taxonomique Erwinia carotovora (Latour et al., 2008). Cette bactérie cosmopolite pathogène de nombreuses plantes cultivées se multiplie en milieu chaud $\left(25^{\circ} \mathrm{C}\right)$ et très humide. Elle nécessite toutefois l'existence de portes d'entrée dans la plante, qui peuvent être des blessures ou bien une perméabilité des lenticelles générée par des excès d'eau asphyxiant les racines (Messiaen et al., 1991)

À notre connaissance, les travaux d'agronomie et de bactériologie sur le céleri branche sont inexistants, notamment en régions tropicales. Un protocole expérimental a donc été mis en place pour étudier l'impact possible du mode de récolte sur le développement de la maladie: l'hypothèse émise était que les blessures du collet occasionnées par la récolte et exposées aux projections de terre favorisaient la propagation de la bactérie. Le mode de récolte échelonnée en feuilles individuelles a été comparé au mode de récolte classique en maraîchage tempéré du céleri branche, qui consiste en l'arrachage de la plante entière.

\section{Protocole expérimental}

Pendant les trois années précédant la mise en place de l'essai (2007), les distributeurs locaux de semences maraîchères ont peu à peu retiré la variété " Plein Blanc Pascal ", très appréciée des producteurs et des consommateurs camerounais, au profit de la variété "Elne ". De ce fait, le protocole expérimental a été installé sur une culture pure de céleri de variété " Elne ", qui était la seule disponible en 2007.

En récolte en plante entière, du fait de laccroissement du nombre de feuilles jusqu'à la récolte, la densité de plantation est basse (20 plants $/ \mathrm{m}^{2}$ ) et inférieure à celle pratiquée pour la récolte échelonnée en feuilles individuelles (30 plants $/ \mathrm{m}^{2}$ ). Notre essai a donc croisé le facteur mode de récolte (feuille versus plante) avec le facteur densité de plantation (basse densité [BD] versus haute densité [HD]), en quatre blocs de Fisher de parcelles élémentaires de $2,5 \mathrm{~m}^{2}$ avec répartition aléatoire des quatre modalités. Le semis réalisé le 31 décembre 2007 a été suivi d'un repiquage le 15 février 2008. La récolte en feuilles individuelles s'est étendue du 26 avril 2008 au 13 août 2008, tandis que la récolte unique en plante entière est intervenue le 25 juin 2008, soit 19 semaines après le repiquage.

\section{Impact du mode de récolte}

Jusqu'à la première récolte en feuilles individuelles, les observations ne révèlent aucun effet de la densité de plantation sur le développement du céleri branche
Résultats et discussion (figures $2 A$ et $2 B$ ). Par la suite, le nombre de feuilles augmente pour les plantes récoltées entières tandis qu'il est quasi constant pendant 8 semaines pour les plantes récoltées en feuilles individuelles, les prélèvements échelonnés ayant été compensés par l'émission de nouvelles feuilles (figure 2A). À partir de la $20^{\mathrm{e}}$ semaine après le repiquage, les

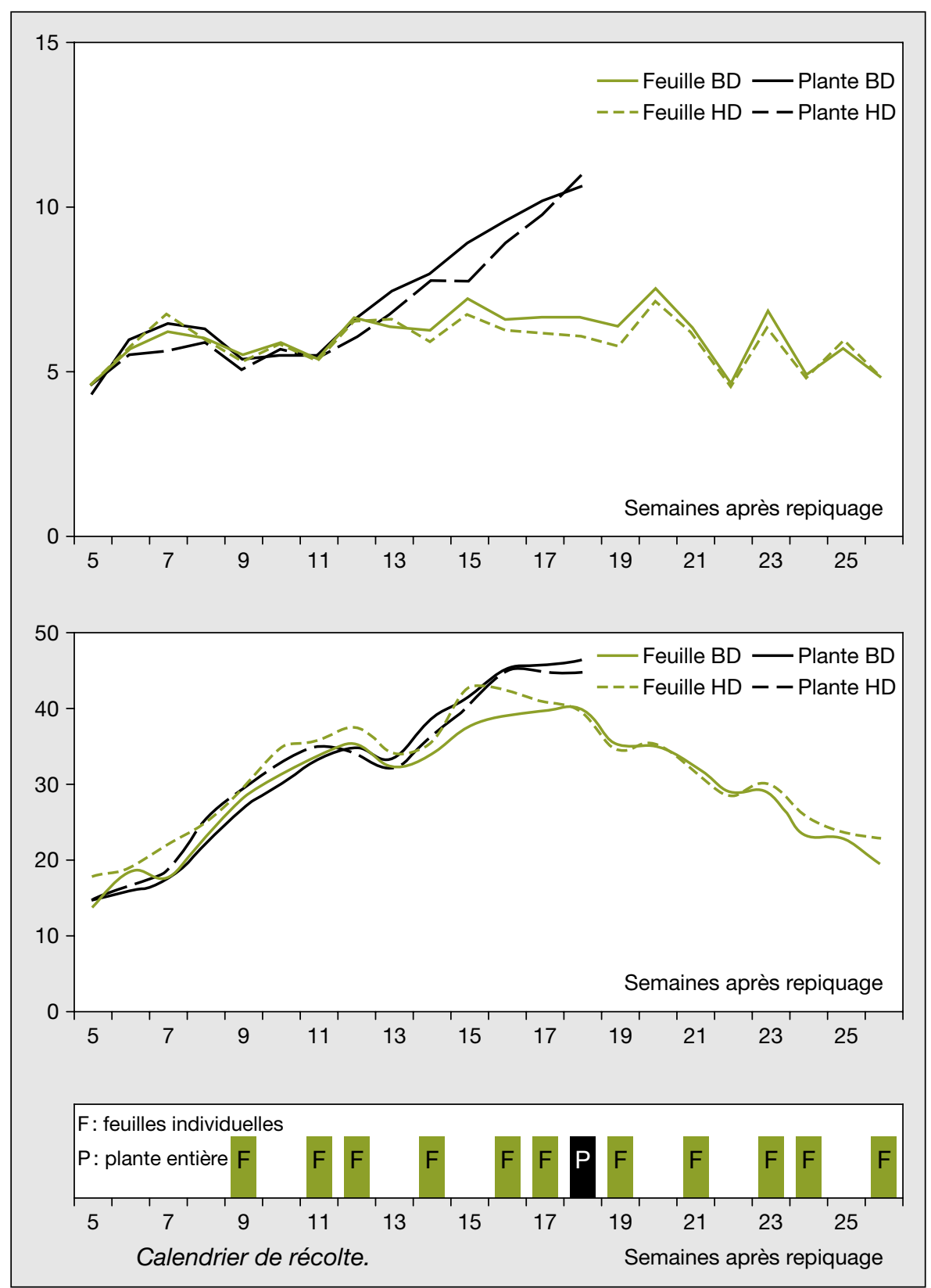

Figure 2. Développement du céleri branche en fonction du mode de récolte (feuille versus plante entière) et de la densité de plantation (basse densité [BD] versus haute densité [HD]).

Figure 2. Growth rate of celery according to the type of harvest (stalk leaf versus whole plant) and planting density (low density [BD] versus high density [HD])

A) évolution du nombre de feuilles en fonction du calendrier de récolte; B) évolution de la longueur des feuilles $(e n \mathrm{~cm}$ ) en fonction du calendrier de récolte. 
plantes récoltées en feuilles individuelles révèlent un épuisement physiologique qui se traduit par une réduction du nombre de feuilles mais aussi par une diminution de la longueur des feuilles (figure $2 B$ ).

Contrairement à l'hypothèse émise, les pourritures se révèlent significativement plus nombreuses en récolte en plante entière (figure 3) : favorisée par les fréquentes irrigations, la dégradation humide des feuilles sénescentes externes induit probablement une occurrence plus forte de la bactérie tandis que la cicatrisation des blessures de récolte en feuilles individuelles en limite la propagation.

Enfin, le rendement en récolte en feuilles individuelles est significativement très supérieur au rendement en récolte en plante entière, respectivement $14,3 \mathrm{~kg} / \mathrm{m}^{2}$ et $3,5 \mathrm{~kg} / \mathrm{m}^{2}(p<0,05)$. En récolte en

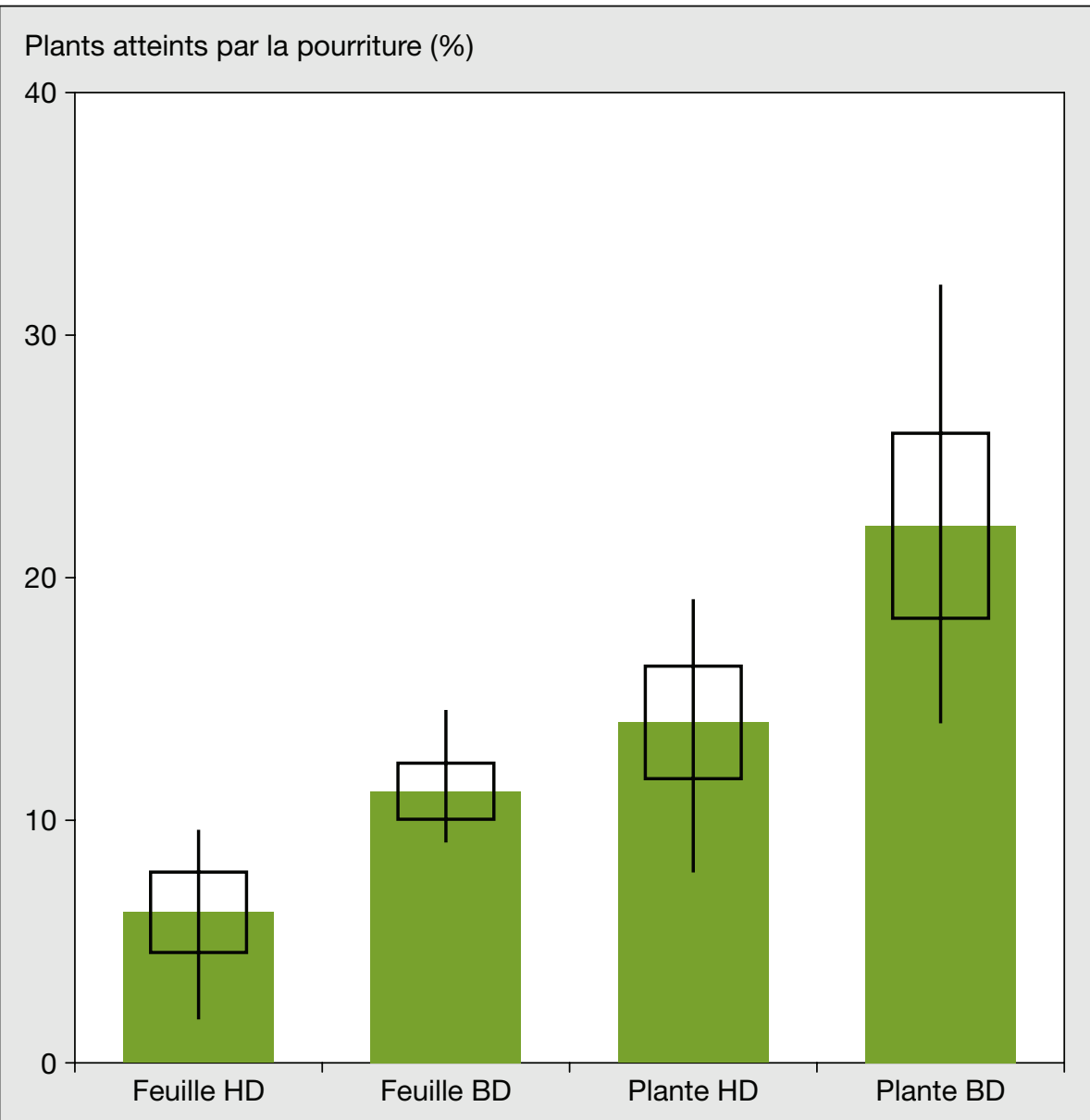

Figure 3. Nombre de plants de céleri branche atteints par la pourriture molle (\% du nombre de plants par parcelle) 19 semaines après repiquage $(p<0,05)$.

Figure 3. Number of celery plants affected by soft decay (\% of plants per plot) 19 weeks after transplanting $(p<0.05)$. feuilles individuelles, l'impact moindre des pourritures est renforcé par un rendement plus élevé par plant : le prélèvement échelonné d'une ou deux feuilles permet une récolte avant que les plants ne soient atteints par la pourriture, alors qu'en récolte en plante entière, les céleris malades sont perdus. La cause de l'augmentation récente des pourritures n'est donc pas liée au mode de récolte en feuilles individuelles.

Par ailleurs, contrairement à la recommandation d'enfouir les amendements organiques pour limiter la volatilisation de l'azote, les maraîchers de Nkolondom épandent en surface une grande quantité de fumier de fientes de volailles $\left(4 \mathrm{~kg} / \mathrm{m}^{2}\right)$. Le couplage de cet amendement avec les irrigations régulières pourrait être favorable au développement de la bactérie. Mais cela ne peut expliquer l'extension récente des pourritures puisque cette pratique a été vulgarisée depuis 1980 à la suite de l'extension des élevages avicoles intensifs autour de Yaoundé.

\section{Autre hypothèse : le changement variétal}

Au Cameroun, depuis la libéralisation du secteur agricole inaugurée à la fin des années 1980, la fourniture des engrais, pesticides et semences aux agriculteurs et maraîchers est assurée par de nombreux distributeurs non spécialisés. Ces revendeurs se sont intéressés en priorité aux grandes cultures d'exportation au détriment d'autres productions, comme les cultures maraîchères, pour lesquelles le marché est plus étroit et l'organisation des producteurs inexistante. C'est ainsi que la variété de céleri branche "Plein Blanc Pascal " a été peu à peu retirée du commerce et remplacée par la variété "Elne ", moins appréciée des producteurs et des consommateurs en raison de ses pétioles plus fins et de son port retombant. La disparition totale d'une variété très appréciée localement, faute de semences commerciales, pose la question de la maîtrise de l'itinéraire technique par les producteurs. Ce fait souligne aussi la vulnérabilité des producteurs lorsque la filière est peu organisée, alors que certains d'entre eux n'hésitent pas à innover pour répondre aux attentes d'un nouveau marché de proximité en pleine expansion. Enfin, cette disparition ne nous a pas permis de mettre en place des essais variétaux participatifs et multilocaux qui auraient eu avantage à être élargis à plusieurs accessions, dans le but d'analyser leurs qualités agronomiques et d'évaluer leur tolérance ou leur résistance à la bactérie Pectobactérium carotovorum.

\section{Conclusion}

La bactérie Pectobactérium carotovorum (anciennement appelée Erwinia carotovora), a été identifiée comme agent causal de la pourriture molle observée sur la culture de céleri branche dans le basfond maraîcher de Nkolondom au Cameroun. L'itinéraire technique, notamment la récolte échelonnée des feuilles, n'explique pas l'extension récente de la maladie. Celle-ci serait plutôt liée à un 
changement variétal imposé récemment par les distributeurs locaux de semences, ce qui illustre la fragilité d'une filière lorsqu'elle est insuffisamment organisée. Ce fait est d'autant plus dommageable que notre étude a montré l'intérêt technico-économique du mode de récolte échelonnée en feuilles individuelles, bien adapté au contexte périurbain local. D'une part, il permet aux ménages à faible pouvoir d'achat, ne possédant en général pas de réfrigérateur, d'acheter au détail les feuilles fraîches de céleri branche correspondant à leur consommation quotidienne et, d'autre part, il procure aux petits producteurs des revenus réguliers pour faire face aux charges d'exploitation mais aussi aux dépenses du ménage.

\section{Remerciements}

Les auteurs remercient Léonard Enama Nga, Jean-Paul Melene et Laurent Nvomo, maraîchers du bas-fond de Nkolondom, pour leur partenariat lors de cette étude.

\section{Références}

Bouzo CA, Pilatti RA, Faravo JC. Control of Blackheart in the Celery (Apium graveolens L.) Crop. J Agr Soc Sci $2007 ; 3$ : 73-4.

Kahane R, Temple L, Brat P, De Bon H. Les légumes-feuilles des pays tropicaux: diversité, richesse économique et valeur santé dans un contexte très fragile. In : Parrot L, ed. Agricultures et développement urbain en Afrique subsaharienne: environnement et enjeux sanitaires. Paris : L'Harmattan, 2008.
Latour X, Faure D, Diallo S, et al. Lutte contre les maladies bactériennes de la pomme de terre dues aux Pectobacterium spp. (Erwinia carotovora). Cah Agric 2008; 17: 355-60. doi : 10.1684/agr.2008.0210

Messiaen CM, Blanchard D, Rouxel F, Lafon R. Les maladies des plantes maraîchères. Paris: Inra éditions, 1991.

Temple L, Marquis S, David O, Simon S. Le maraîchage périurbain à Yaoundé est-il un système de production localisé innovant? Economies et Sociétés, Ser Systèmes agroalimentaires 2008 . $30: 2309-28$.

Van Rensburg WJ, Venter SL, Netshiluvhi TR, van den Heever E, Vorster HJ, de Ronde JA. Role of indigenous leafy vegetables in combating hunger and malnutrition. S Afr J Bot 2004 ; $70: 52-9$. 